\title{
Ultrasound Evaluation of Uterine Leiomyoma in Perimenopausal Females with Histopathological Correlation
}

\author{
Sakshi Rajendra Daga ${ }^{1}$, Suresh V. Phatak ${ }^{2}$ \\ ${ }^{1}$ Department of Radiodiagnosis, JNMC, Sawangi (Meghe), Wardha, Maharashtra, India. \\ ${ }^{2}$ Department of Radiodiagnosis, JNMC, Sawangi (Meghe), Wardha, Maharashtra, India.
}

\section{ABSTRACT}

\section{BACKGROUND}

Abnormal uterine bleeding remains an important health issue in perimenopausal females, often a cause of high morbidity and mortality. Uterine leiomyoma is the major cause of abnormal or irregular bleeding. We wanted to evaluate the role of ultrasound in assessing uterine leiomyomas and determine its sensitivity, specificity and diagnostic accuracy in comparison to histopathological studies.

\section{METHODS}

This is a cross sectional, observational and analytical study conducted in the Department of Radiodiagnosis at AVBRH, (Sawangi Meghe), a rural tertiary care hospital in central India. The study was carried out over a period of 6 months from January 2019 to June 2019. 50 patients in perimenopausal age group of 40-50 years were selected on the basis of predetermined inclusion and exclusion criteria. All included patients were counselled and asked for informed consent before entry into the study. Statistical analysis was done using descriptive and inferential statistics using sensitivity, specificity, positive predictive value, negative predictive value, and diagnostic accuracy.

\section{RESULTS}

Most of the uterine leiomyomas were intramural in location and hypoechoic in echotexture. Intramural fibroid was the most common fibroid detected constituting $88 \%$. The sensitivity, specificity, positive predictive value [PPV], negative predictive value [NPV], and diagnostic accuracy of ultrasound in correlation to histopathology was found to be $93.75 \%, 91.67 \%, 97.83 \%, 78.57 \%$ and $93.33 \%$ respectively.

\section{CONCLUSIONS}

Ultrasound has high sensitivity and specificity in diagnosing the uterine leiomyomas. It should be used as primary investigative modality for work up of abnormal uterine bleeding in perimenopausal age group.

\section{KEY WORDS}

Perimenopausal, Ultrasound, Fibroid, Histopathological.
Corresponding Author: Dr. Sakshi Rajendra Daga, Department of Radiodiagnosis, JNMC, AVBRH, Sawangi Meghe, Wardha-442001, Maharashtra, India. E-mail: sakshidaga1991@gmail.com

\section{DOI: $10.14260 / \mathrm{jemds} / 2020 / 125$}

Financial or Other Competing Interests: None.

How to Cite This Article:

Daga SR, Phatak SV. Ultrasound evaluation of uterine leiomyoma in perimenopausal females with histopathological correlation. J. Evolution Med. Dent. Sci. 2020;9(08): 562-565, DOI: 10.14260/jemds/2020/125

Submission 18-12-2019,

Peer Review 29-01-2020,

Acceptance 05-02-2020,

Published 24-02-2020.

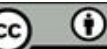




\section{BACKGROUND}

In the perimenopausal age group, menstrual disorders are the most common reason for frequent gynaecologist visits. ${ }^{[1]}$ Abnormal uterine bleeding [AUB] is defined as "bleeding that results apart from normal menstrual cycle or irregular menstrual bleeding that may be heavier, prolonged or frequent.[1] Patients with abnormal uterine bleeding constitute around $5-10 \%$ of cases visiting the outpatient gynecological clinic. It is estimated that around $50 \%$ lie in the age group of 40-50 years.[2] As women approaches towards the menopause, she may experience changes in normal menstrual cycle due to hormonal shifts or fluctuations during this phase resulting in varied menstrual patterns or irregularities like heavier and longer periods, less frequent cycles, intermenstrual spotting etc.[3] Uterine fibroids are one of the leading causes of abnormal uterine bleeding in this age group. They are regarded as the most prevailing benign uterine neoplasms. They are derived from smooth muscles with intervening fibrous tissue within. ${ }^{[4]}$ Based on site of origin they can be further classified as intramural (those arising from myometrium), submucosal (those noted within the endometrium) and subserosal (the ones lying beyond serosal layer of uterus). The most common fibroids are myometrial or intramural. Submucosal fibroids are although rare but present with severe bleeding. ${ }^{[5]}$ Some of the fibroid may exhibit haemorrhagic foci, necrotic degeneration or calcification within while in rare instances few of them may have fatty content within.[6]

Gynecologists are often unable to recognize the cause or etiology of abnormal menstrual bleeding despite of proper history and clinical examination. Newer Diagnostic modalities and techniques are emerging over time thereby helping gynecologists to plan an appropriate treatment algorithm for patients with menstrual ailments. Previously dilatation and curettage were considered as investigation of choice to diagnose an intrauterine lesion. It is an invasive procedure performed under anesthesia, at times becomes risky in patients with recurrent bleeding. [7] The current modalities for evaluation of abnormal bleeding include hysteroscopy and ultrasound. Hysteroscopy is an endoscopic visualization of uterine cavity and cervical canal. It can determine exact location of intrauterine pathology. The major drawback of this technique is requirement of anesthesia, invasiveness and lack of affordability.[8] [9] The advent and use of diagnostic ultrasound have proved beneficial in assessing pathologies like fibroids which are one the major cause of uterine bleeding. Ultrasound serves as first line imaging modality for precise evaluation of size, number and location of fibroid, thereby making it possible to plan appropriate treatment algorithm for patients.[10]

Our hospital caters to a large rural population, several of whom are females with complaints of perimenopausal bleeding. It remains an important health issue in females, often a cause of high morbidity and mortality. Ultrasonography plays a pivotal role in the assessment of abnormal uterine bleeding due to its wide availability, good resolution, low cost and no risk of radiation exposure. The aim of our study was to find out role of ultrasound in detection of uterine leiomyomas among 50 perimenopausal females who presented to gynaecological outpatient department with complaints of abnormal uterine bleeding and determining sensitivity and specificity of ultrasound in correlation to histopathological study.

\section{METHODS}

This study was carried out in department of Radio diagnosis, Acharya Vinobha Bhave Rural Hospital, Sawangi Wardha, a tertiary care rural health hospital. It was a cross sectional form of study conducted over a span 6 months (January 2019 -June 2019). The sample size for this study was selected on the basis of predefined exclusion and inclusion criteria. Study population included 50 perimenopausal females in 40-50 years of age group who visited gynaecology clinic with complaints of abnormal menstrual bleeding or alteration in menstrual cycles. Patients with abnormal bleeding in other age groups that is before 40 years and beyond 50 years were excluded. Those not giving consent for study, post-operative patients and those undergoing treatment for other gynaecological malignancy were also excluded.

Ethical clearance was obtained from the institution. Whole procedure was explained to the patient prior to the beginning of ultrasound examination and consent was obtained from them. A female attendant was always present with patient during ultrasound examination. Transabdominal scan was performed with full bladder using 3-5 $\mathrm{MHz}$ convex probe and wherever needed transvaginal scan with empty bladder was obtained using transvaginal probe of $5-11 \mathrm{MHz}$ frequency. Patient was asked to lie down comfortably in a supine position. Transabdominal probe was placed in the suprapubic area over the bladder and was caudally angled to obtain longitudinal section of uterus, cervix and vagina. Then the orientation was changed to transverse section by angling the probe. While doing so, vaginal walls, cervix and body of uterus were studied. For transvaginal scan, the patient was placed in the lithotomy position after having emptied her bladder. Transvaginal probe was covered with sterile latex condom and secured by a rubber band in order to prevent cross-contamination. Before the condom was pulled over the shaft of the probe, a small amount of acoustic gel was inserted inside the tip of the condom. Further to facilitate the probe insertion, it was coated with the acoustic gel. The transducer was inserted into the vagina. Initially, a longitudinal scan was done followed by transverse scan.

Ultrasound findings were noted in terms of size, shape, location, echogenicity of fibroid was noted. Vascularity was assessed with colour Doppler. Patients subsequently underwent Dilatation \& Curettage [D and C] or endometrial biopsy or Hysterectomy procedure as indicated by the gynaecologists. These tissue specimens were then sent to the Histopathology section of Department of Pathology. Later on, tissue processing was done followed by routine haematoxylin and eosin staining. Ultrasound finding were confirmed by histopathology.

\section{Statistical Analysis}

Statistical analysis was done using descriptive and inferential statistic's using sensitivity, specificity, positive predictive value [PPV], negative predictive value [NPV] and diagnostic accuracy as statistical parameters. 


\section{RESULTS}

Majority of the fibroids were found to be intramural in location accounting for 44 cases $(88 \%)$ followed by submucosal fibroids.(8) (table 1). Only 2 fibroids (4\%) were found to have subserosal location. The most common fibroid that is intramural fibroid on ultrasound appeared as a welldefined hypo/hype/iso or mixed echogenicity lesion, located either in anterior or posterior myometrial wall exhibiting posterior shadowing. On ultrasonography, maximum fibroids appeared hypoechoic in appearance constituting 78\% followed by heterogenous appearance (10\%). (table 2)

\begin{tabular}{|c|c|c|}
\hline Echogenicity of Fibroid & Number & Percentage \\
\hline Hypoechoic & 39 & $78 \%$ \\
\hline Isoechoic & 4 & $8 \%$ \\
\hline Hyperechoic & 2 & $4 \%$ \\
\hline Mixed echogenicity (heterogenous) & 5 & $10 \%$ \\
\hline Total & 50 & $100 \%$ \\
\hline
\end{tabular}

\begin{tabular}{|c|c|c|}
\hline Location of Fibroids & Number & Percentage \\
\hline Intramural & 44 & $88 \%$ \\
\hline Submucosal & 4 & $8 \%$ \\
\hline Subserosal & 2 & $4 \%$ \\
\hline \multicolumn{2}{|c|}{ Table 2. Ultrasound Appearance of Fibroids } \\
\hline
\end{tabular}

\begin{tabular}{|c|c|c|c|c|c|c|c|c|c|}
\hline & \multicolumn{2}{|c|}{$\begin{array}{c}\text { Histopathological } \\
\text { Diagnosis }\end{array}$} & \multirow[b]{2}{*}{ 푱 } & \multirow{2}{*}{ 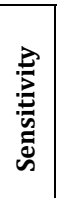 } & \multirow{2}{*}{ 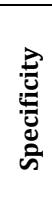 } & \multirow[b]{2}{*}{$\frac{a}{a}$} & \multirow[b]{2}{*}{$\frac{2}{z}$} & \multirow[b]{2}{*}{ 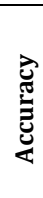 } \\
\hline & & 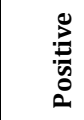 & 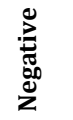 & & & & & & \\
\hline Ultrasound & Positive & 35 & 1 & 36 & \multirow{2}{*}{ خें } & \multirow{2}{*}{$\begin{array}{l}\text { مْ } \\
\text { ف } \\
\text { a }\end{array}$} & \multirow{2}{*}{ సิ̀ } & \multirow{2}{*}{ 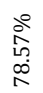 } & \multirow{2}{*}{ ईे } \\
\hline \multicolumn{2}{|c|}{ Total } & $\frac{3}{38}$ & $\begin{array}{l}11 \\
12\end{array}$ & $\begin{array}{l}14 \\
\mathbf{5 0}\end{array}$ & & & & & \\
\hline
\end{tabular}

Out of 50 ultrasound detected fibroids. 35 were confirmed on histopathology. Hematoxylin \& Eosin stained section confirmed findings of fibroid showing smooth muscle bundle arranged in fascicles or whorls and are separated by intervening fibrovascular stroma in between. The sensitivity of ultrasound in detecting fibroid was found to be $92.11 \%$. Specificity was $91.67 \%$, the positive predictive value (PPV) was $97.22 \%$ and negative predictive value (NPV) was $78.57 \%$. Ultrasound had a diagnostic accuracy of $92 \%$. (table 3).

\section{DISCUSSION}

A woman in a phase of menopause transition may experience varying symptoms during this period such as flushes or sweating, mood swings, anxiety, sleeping disturbance, etc. One such symptom includes abnormal uterine bleeding which may occur in the form of heavy bleeding, heavy and prolonged menstrual cycle, irregular or intermenstrual bleeding, etc. Abnormalities in menstrual patterns may lead to significant debilitating health problems in females. Uterine fibroids have attributed to major cause of bleeding in perimenopausal period. Thorough evaluation of these females through proper clinical examination and imaging modalities like ultrasonography plays an instrumental and decisive role in finding out the exact cause of abnormal bleeding, thereby aiding gynaecologists and surgeons to plan an appropriate treatment and surgical intervention if required. This study aims at evaluating fibroids or leiomyomas through ultrasound modality and correlating them with histopathological findings. In the present study, out of 50 ultrasound detected fibroids, majority of the fibroids were found to be intramural in location comprising $88 \%$ followed by fibroids in submucosal region (8\%). Similar findings were reported by Sarkodie BD et al. [11] who carried out a study on Ghanaian women and evaluated ultrasound characteristics and patterns of uterine fibroids. He found a predominance of intramural fibroids comprising 57.8\%. Subserosal fibroid were reported to be second most common type of leiomyomas in his study. Study conducted by Handa K et al. on ultrasound evaluation of uterine fibroids reported predominance of intramural fibroids followed by submucosal fibroids.[12]

Fibroid on ultrasonography appear as a well-defined, rounded, usually hypoechoic lesion, which can be isoechoic or hypoechoic to myometrium with some lesions showing calcification and areas cystic degeneration within. In the present study maximum fibroids appeared hypoechoic on ultrasonography accounting for $78 \%$ followed by heterogenous appearance of constituting $10 \%$ all fibroids. Findings were coherent with study conducted by Handa K et al. 93.75\%, 91.67\%, 97.83\%, 78.57\% and $93.33 \%$ respectively. During pathological study of tissue specimen, Hematoxylin and Eosin [H and E] stained section showed smooth muscle cells arranged in whorl pattern with intervening fibrovascular stroma suggestive of fibroid. In the present study it was observed that the sensitivity, specificity, positive predictive value and negative predictive value of sonography for intrauterine fibroid was found to be. $93.75 \%$, $91.67 \%, 97.83 \%$ and $78.57 \%$ respectively when compared to histopathological reports. The diagnostic accuracy of ultrasound was found to be Talukdar B et al. [13] studied the sensitivity, specificity, positive predictive value, negative predictive value of uterine fibroids which was $89.13 \%$, $89.47 \%, 87.23 \%, 91.07 \%$ respectively, which was similar to our study. Study conducted by Hanafi M et al. [14] showed sensitivity, specificity, positive predictive value and negative predictive value of fibroid was 96.38\%,96\%,99.25\%and $82.76 \%$ respectively. These findings are concordant with our present study. Dipi RM et al. [15] showed that sensitivity, specificity, positive predictive value, negative predictive value of transabdominal sonogram for uterine fibroid was $88.9 \%, 88.6 \%, 80 \%$ and $93.9 \%$ respectively while that of Transvaginal sonography [TVS] was $94.9 \%, 91.4 \%, 85 \%$ and $97 \%$ respectively.

\section{Limitations}

Obscuration of pelvic organs due to bowel gases affects proper visualisation of organ as well as alters image quality, limited or restricted penetration of ultrasound in fat or obese patients. Ultrasound has a restricted field of view which creates difficulty in analysing the site of lesion and the technique of ultrasound depends upon the skills of an operator resulting in interobserver variability. 


\section{CONCLUSIONS}

Ultrasound plays a pivotal role in diagnosing the leiomyoma as the cause of abnormal uterine bleeding in perimenopausal females. Ultrasound, especially transabdominal, serves as a first line investigative modality in females who suffer from excessive bleeding by offering the advantage of being noninvasive. Moreover, it is cost effective, safer, and widely accessible. It aids gynaecologists and surgeons by providing detailed information about the location, size, characteristics and vascularity of the fibroids and also determines its relation to adjoining pelvic organs. This helps in formulating a suitable medical or surgical management workup for the patients. Though it cannot substitute histopathology which is considered as the gold standard but definitely improvises detection of fibroids or leiomyoma and contributes by reducing false positive results. Better diagnostic accuracy of fibroid on ultrasound diminishes invasive procedures in patients. It is a non-ionising and affordable modality for patients. Ultrasound is a best diagnostic tool in developing countries like India where majority of the population is rural based and cannot afford the cost of CT and MRI.

\section{REFERENCES}

[1] Kjerulff KH, Erickson BA, Langenberg PW. Chronic gynecological conditions reported by US women: findings from the National Health Interview Survey, 1984 to 1992. American Journal of Public Health 1996;86(2):195-9.

[2] Rezk M, Masood A, Dawood R. Perimenopausal bleeding: patterns, pathology, response to progestins and clinical outcome. Journal of Obstetrics and Gynaecology 2015;35(5):517-21.

[3] Dalal PK, Agarwal M. Postmenopausal syndrome. Indian Journal of Psychiatry 2015;57(Suppl 2):S222-32.

[4] Prayson RA, Hart WR. Pathologic considerations of uterine smooth muscle tumors. Obstetrics and Gynecology Clinics of North America 1995;22(4):637-57.

[5] Murase E, Siegelman ES, Outwater EK, et al. Uterine leiomyomas: histopathologic features, MR imaging findings, differential diagnosis and treatment. Radiographics 1999;19(5):1179-97.

[6] Ueda H, Togashi K, Konishi I, et al. Unusual appearances of uterine leiomyomas: MR imaging findings and their histopathologic backgrounds. Radiographics 1999;19(Spec. No):S131-45.

[7] Bakos 0, Heimer G. Transvaginal ultrasonographic evaluation of the endometrium related to the histological findings in pre- and perimenopausal women. Gynecologic and Obstetric Investigation 1998;45(3):199204.

[8] Kotdawala P, Kotdawala S, Nagar N. Evaluation of endometrium in peri-menopausal abnormal uterine bleeding. Journal of Mid-Life Health 2013;4(1):16-21.

[9] Emanuel MH, Verdel MJ, Wamsteker $\mathrm{K}$, et al. A prospective comparison of transvaginal ultrasonography and diagnostic hysteroscopy in the evaluation of patients with abnormal uterine bleeding: clinical implications. American Journal of Obstetrics and Gynecology 1995;172(2 Pt 1):547-52.

[10] Woźniak A, Woźniak S. Ultrasonography of uterine leiomyomas. Przeglad Menopauzalny 2017;16(4):113-7.

[11] Sarkodie BD, Botwe BO, Ofori EK. Uterine fibroid characteristics and sonographic pattern among Ghanaian females undergoing pelvic ultrasound scan: a study at 3major centres. BMC Women's Health 2016;16(1):10.

[12] Handa K, Tayade A, Kale S, et al. Evaluation of uterine fibroids as a cause of AUB by pelvic ultrasound. International Journal of Biomedical and Advance Research 2016;7(3):107-12.

[13] Talukdar B, Mahela S. Abnormal uterine bleeding in perimenopausal women: correlation with sonographic findings and histopathological examination of hysterectomy specimens. Journal of Mid-Life Health 2016;7(2):73-7.

[14] Hanafi M. Ultrasound diagnosis of adenomyosis, leiomyoma or combined with histopathological correlation. Journal of Human Reproductive Sciences 2013;6(3):189-93.

[15] Dipi RM, Amin MS, Islam MN, et al. Comparison of transabdominal and transvaginal sonography in the evaluation of uterine mass with histopathological correlation. Mymensingh Medical Journal 2013;22(1):69-74. 